Cuadernos de Estudios Empresariales

ISSN: 1131-6985

http://dx.doi.org/10.5209/CESE.57369

\title{
Financiación de micro y pequeñas empresas comercializadoras locales
}

\author{
Jazmín Díaz-Barrios $^{1}$; Claudia Velazco ${ }^{2}$
}

Recibido: / Aceptado:

Resumen. El estudio se dirigió a analizar la financiación de micro y pequeñas empresas comercializadoras de suministros industriales bancarizadas en el Municipio Maracaibo, Zulia, Venezuela. Investigación descriptiva, diseño no experimental transversal de campo. Se encontró que este sector está conformado por microempresas familiares, con altas ventas, dirigidas por personal profesional. A corto plazo se financian con crédito comercial y pasivos acumulados, poco bancario. A largo plazo, no utilizan financiación externa. Sobre el capital de trabajo, financian activos circulantes con pasivos circulantes y permanentes. Como estrategias, aceleran cobros, retardan pagos y aprovechan descuentos por pronto pago. Se concluye que, estas organizaciones son bastante cerradas a influencias externas, hasta ahora han preferido mantenerse con un control financiero casi total, sin apalancamiento, lo cual a su vez limita su expansión. No utilizan técnicas de evaluación, indicadores o razones financieras; las decisiones financieras son familiares. Su alta rentabilidad en contraste con su pequeño tamaño, les ha reforzado las prácticas tradicionales. Por otro lado, la alta incertidumbre legal, social y económica que vive el país los orienta hacia un horizonte de muy corto plazo, sin expectativas. Aun cuando esta investigación no se puede generalizar al sector, muestra que el bajo uso de crédito bancario es un problema de ausencia de demanda y no de falta de oferta; por otra parte señala que en empresas familiares, las decisiones no obedecen a criterios técnicos, a pesar de que tengan personal preparado.

Palabras clave: Empresas comercializadoras; Estrategias de financiación; Mipyme; Suministros industriales.

\section{[en] Financing micro and small local trading companies}

Abstract. This study aimed to analyze the small and medium industrial supply distribution companies' financing strategies in the Maracaibo municipality. It was field, descriptive, cross-sectional, non-experimental research. It was established that this sector is dominated by family businesses, featuring high sales, led by professional staff. In the short-term, they use internal and external financing. In long-term, they do not use external financing. As for working capital, current assets are financed with current liabilities and also with permanent liabilities. As strategies, accelerated collections, delayed payments and taking advantage of discounts for prompt payments. In conclusion, these organizations are quite closed to external influences; so far they have preferred to maintain almost complete financial control, without leveraging, which in turn limits their expansion. They do not use financial evaluation techniques, indicators, or financial ratios. Their high profitability, compared with their small size, has reinforced traditional practices. On the other hand, the high legal, social, and economic, uncertainty in the country, guides them towards a very short-term horizon, without expectations. Even if this research cannot be generalized to the industry at large, it showcases how the low usage rate of the bank system follows a lack of demand rather than an absence of supply. On the other hand,

\footnotetext{
Universidad del Zulia

jazmin.diaz@fces.luz.edu.ve

2 SIAPCA-Suministros industriales

info@siapca.com
} 
it suggests that in family-owned businesses, decisions do not follow technical criteria, even if they have staff with financial expertise.

Key words: Commercial companies; industrial supplies; SMEs; strategies for financing.

Sumario: 1. Introducción. 2. Aspectos metodológicos. 3. Aspectos teóricos relevantes. 4. Resultados. 5. Conclusiones.

Cómo citar: Díaz-Barrios, J.; Velazco, C. (2017). Financiación de micro y pequeñas empresas comercializadoras locales, Cuadernos de Estudios Empresariales, 27, 27-42.

\section{Introducción}

De acuerdo con el Banco Interamericano de Desarrollo (BID, 2012) y Ferraro (2011) de la, existen grandes obstáculos para financiar a las Pequeñas y medianas empresas (Pymes), siendo el principal de ellos la informalidad con que operan estas empresas; además, los bancos perciben que representa un alto riesgo por la falta de información confiable, el costo administrativo interno y las trabas relacionadas con políticas de los gobiernos, especialmente las regulaciones financieras. En este mismo orden de ideas, Bloch y Granato (2007), expresan que la oferta de crédito a Pymes se caracteriza por altas tasas de interés (el doble que para las grandeslalo mencionado por el BID. Pareciera que las condiciones de acceso al crédito para las Pymes son poco competitivas en los países de la región, aun cuando el mismo informe del BID (2012) reporta que ha incrementado el crédito de la banca a pymes.

Afirmaban Pollack y García (2004) que aunque el acceso al crédito en América Latina alcanzaba niveles comparables con los países industrializados, en la mayoría de los países había indicios de que existían proyectos Pymes que se dejaban de financiar por las altas tasas y consecuentemente, la necesidad de tasas de retorno interno elevadas para rentabilizarlos. En el caso venezolano, plantean Díaz y Piña (2013) que son grandes los esfuerzos por disminuir las barreras existentes, pero que éstas son inmensas. Entre ellas se pueden citar algunas sumamente perjudiciales para las pymes venezolanas, tales como recursos tecnológicos no competitivos con el primer mundo (flexibilidad, producción a escala, producción automatizada), recurso humano que no está suficientemente preparado para operar tecnología (y los empresarios no se ocupan en capacitarlos), además de las barreras financieras mencionadas.

En función de lo antes mencionado, se consideró pertinente realizar un estudio enfocado en analizar las estrategias de financiación de las pymes en el entorno venezolano en general y zuliano en particular. En primer término se caracterizó el sector seleccionado, para luego revisar las fuentes de financiación utilizadas, la estructura y las estrategias en uso actualmente, finalmente se presentaron algunas conclusiones basadas en los hallazgos. 


\section{Aspectos metodológicos}

Metodológicamente, el estudio tuvo un alcance descriptivo, de campo, no experimental, transversal descriptivo, con un abordaje cuantitativo. Para definir la población, se consideraron los conceptos de universo, población y unidades de observación, de acuerdo con Parra (2006). El universo son las micro, pequeñas y medianas empresas (Mipymes) comercializadoras de suministros industriales bancarizadas situadas en la ciudad de Maracaibo, municipio Maracaibo, estado Zulia, Venezuela. En atención a lo anterior, la población definida fueron las mipymes comercial de suministros industriales clientes del Banco Banesco, Agencia Paraíso, Maracaibo, lo cual da una población de 20 organizaciones, de acuerdo a listado recibido de la gerencia de la agencia en cuestión.

No se seleccionó muestra, ya que el instrumento se aplicó a las 20 empresas recibidas en el listado del banco, lo cual se corresponde con un censo de acuerdo con Hernández et al. (2010). Como unidades de observación, se consideraron los gerentes de finanzas (o quien desarrollara esta actividad) en cada empresa.

Los instrumentos de recolección de datos fueron la revisión documental y un cuestionario auto-administrado de 48 preguntas, validado por cinco expertos y con una confiabilidad de $\mathrm{rtt}=0.9596$. Para tales fines, se aplicó a diez (10) gerentes de finanzas de otras empresas con similares características, el instrumento en dos oportunidades, separadas por un lapso de 7 días, a fin de utilizar el método test-retest y correlacionar los resultados de la primera y segunda aplicación con el Coeficiente de Estabilidad y Equivalencia, recomendado cuando se trata de un cuestionario mixto, con opciones de respuestas dicotómicas, de selección múltiple y de simple escogencia (Chávez, 2006).

Para el tratamiento de la data se construyeron matrices para la tabulación y se aplicó estadística descriptiva: distribuciones de frecuencia absolutas (F) y relativas (\%) de las respuestas emitidas en el instrumento del estudio.

\section{Aspectos teóricos relevantes}

Podría definirse la financiación como el conjunto de recursos monetarios financieros requeridos para llevar a cabo una actividad económica determinada. Está compuesto por los recursos propios, y complementado con recursos de tercero recibidos en préstamo u otras modalidades. Cuando un individuo u organización define la financiación que requiere para un proyecto o inversión determinada, lo hace basado en algunas teorías de estructura financiera que existen al respecto como la teoría de Pecking Order, Trade off o el teorema de Modigliani-Miller, por citar algunos (Herrera y col., 2005). Ahora bien, estas teorías definen, de alguna manera, la forma de actuación de los gerentes en las organizaciones y a partir de ellas se precisa la estructura de financiación que elegirán en una circunstancia determinada.

\section{a) Estructura de financiación}

Según Rubio (2006), la estructura de financiación es la proporción entre patrimonio y deuda que la empresa utiliza para financiar sus activos (proyectos). Básicamente 
una empresa puede elegir entre tres formas de financiación: emitir más capital, incurrir en deuda, o utilizar las utilidades retenidas. Existen algunas ventajas en tener deudas. El beneficio principal está ligado a los impuestos, ya que los intereses se deducen antes de determinar los beneficios gravables con impuestos y así, la deuda se hace menos onerosa comparada con el patrimonio. Pero, existen desventajas de tener deuda, principalmente el peligro de la quiebra y el hecho de tener que reportar a terceros fuera de la empresa.

El administrador de empresas responsable debe elegir una estructura de financiación que equilibre tales costos y beneficios. Esto debe hacerse analizando las particularidades del negocio y las preferencias en cuanto a la adecuada elección riesgo-retorno que desee asumir como también su preferencia por compartir las decisiones. De acuerdo con Álvarez (2008), la elección de una estructura de financiación genera dos tipos de riesgos: 1) el riesgo vinculado a la actividad que desarrollará la empresa y 2) el riesgo financiero que surge de la elección de la fuente de financiación. Los aumentos de rentabilidad están asociados a los niveles de riesgo, por lo que la estructura de financiación conlleva la necesidad de asumir riesgos.

De acuerdo con Briozzo y Vigier (2006), la decisión de estructura de capital abarca dos dimensiones: fuente y duración. Al decidir la fuente de financiación, se debe elegir entre capital propio -interno o externo- y deuda, que puede provenir de diversas fuentes. Las fuentes internas o autofinanciación son los generados por el funcionamiento del negocio: las amortizaciones y las utilidades retenidas. Entre las fuentes externas de capital propio, se encuentran el aporte de los propietarios e incorporación de nuevos socios, en las empresas de capital cerrado. En las empresas de capital abierto, emisión de acciones.

En cuanto al capital ajeno, las fuentes externas de deuda se pueden clasificar en tres grupos: Instituciones financieras (préstamos de bancos comerciales y otras instituciones financieras); instituciones no financieras y Estado (Pasivos operativos como crédito comercial, créditos de otros negocios, salarios, cargas sociales, tasas e impuestos a pagar).

Ahora bien, cuando se habla de la estructura financiera, se toman decisiones sobre la proporción de la financiación con el cual existirá una organización empresarial. Sin embargo, adicionalmente, es importante definir la temporalidad de esa financiación. Es decir, cuando, en qué cuantía, por qué y para qué serán requeridos fondos a corto o a largo plazo. Aybar y col (2001) indican que es necesario contar con un método que determine la estructura financiera porque el valor de la empresa depende de ello.

\section{b) Financiación a corto plazo}

Según Ross y col. (2005) la financiación a corto plazo es el análisis de las decisiones que inciden en los activos circulantes y los pasivos circulantes, cuyos efectos suelen reflejarse en la empresa en el lapso de un año. Considerando estos aspectos, de acuerdo a Higuerey (2004), se tiene la clasificación de las fuentes de financiación a corto plazo, tal como lo muestra el cuadro 1. 
Cuadro 1. Fuentes de Financiación a Corto Plazo

\begin{tabular}{|c|c|c|}
\hline Tipo & \multicolumn{2}{|l|}{ Fuente } \\
\hline \multirow{9}{*}{$\begin{array}{l}\text { Financiación } \\
\text { sin garantía }\end{array}$} & \multirow{3}{*}{ Fuentes Espontáneas } & Cuentas por pagar \\
\hline & & Pasivos acumulados \\
\hline & & Documentos de crédito \\
\hline & \multirow{2}{*}{ Fuentes Bancarias } & Línea de crédito \\
\hline & & Crédito revolvente \\
\hline & \multirow{4}{*}{ Fuentes Extraordinarias } & Papel commercial \\
\hline & & Carta de crédito \\
\hline & & Anticipos de clients \\
\hline & & Préstamos privados \\
\hline \multirow{2}{*}{$\begin{array}{l}\text { Financiación con } \\
\text { garantía }\end{array}$} & \multicolumn{2}{|l|}{ Inventario } \\
\hline & \multicolumn{2}{|l|}{ Cuentas por cobrar } \\
\hline
\end{tabular}

Fuente: Elaboración propia a partir de Higuerey (2004).

Dentro de la clasificación de fuente de financiación a corto plazo, se establecen las financiaciones con y sin garantías. Según Van Horne y Wachowicz (2002), con garantía, es una forma de deuda en la que se caucionan determinados activos para garantizar el pago. Las garantías que se dan, por lo general son activos como: Cuentas por Cobrar e Inventarios.

El financiación sin garantía, es una forma de deuda que no tiene respaldo de una garantía integrada por determinados activos. Van Horne y Wachowicz (2002). mencionan que el financiación a corto plazo se puede clasificar dependiendo de si su fuente es espontánea o no. Las cuentas por pagar y los gastos acumulados se consideran espontáneos porque se derivan de manera natural de las transacciones diarias. Su magnitud es básicamente una función del nivel de operaciones de las empresas. Conforme a éstas se expanden, por lo general tales pasivos se incrementan y se utilizan para financiar parte de la acumulación en activos.

Hernández y Col (2001) encontraron en su estudio que las cuentas por pagar a proveedores constituyen la principal fuente de financiación a corto plazo empleada por las pequeñas y medianas empresas, explicando que se debe a las facilidades para su otorgamiento en términos de los requisitos exigidos y el período de aprobación, lo que implica según Van Horne y Wachowicz (2002), que la decisión con respecto al crédito se dé con relativa celeridad.

Los mismos autores afirman que las fuentes de financiación a corto plazo, el préstamo bancario se constituye en la vía más expedita para la obtención de los recursos monetarios necesitados por los pequeños y medianos establecimientos para capital de trabajo, compra de maquinaria y equipos, y nuevas inversiones, siendo considerada una modalidad viable a través del pagaré convencional y la línea de crédito. 
Adicionalmente, los bancos tienen el Crédito revolvente, el cual según Van Horne y Wachowicz (2002), es un compromiso formal y legal que asume un banco de extender crédito hasta cierto límite. Mientras dicho compromiso esté vigente, la institución deberá extender crédito siempre que el prestatario desee un préstamo, siempre y cuando que éstos últimos no rebasen la suma especificada.

Dentro de las Fuentes de Crédito Extraordinarias Higuerey (2006) plantea los siguientes tipos: El papel comercial (tipo de pagaré sin protección que emiten las empresas grandes y sólidas y que venden principalmente a otras, a aseguradoras, a fondos de pensiones, a fondo mutualistas del mercado de dinero y a los bancos, con el fin de obtener recurso de una forma más barata, Brigham y Houston, 2006). Además, está la carta de crédito (herramienta común para compras entre empresas sin experiencia mutua o distantes geográficamente); el anticipo de clientes (modalidad de financiación económico y sin garantía) y los préstamos privados, hechos por accionistas de gran patrimonio que pueden prestar dinero a la empresa a fin de solventar una situación determinada.

\section{c) Financiación a largo plazo}

De acuerdo con Vera (2009), la financiación a largo plazo proviene de diferentes tipos de fuentes, las cuales se resumen en el cuadro 2.

Cuadro 2. Fuentes de Financiación a Largo Plazo

\begin{tabular}{|l|l|l|}
\hline \multicolumn{2}{|c|}{ Tipo } & \multicolumn{1}{c|}{ Fuente } \\
\hline \multirow{2}{*}{$\begin{array}{l}\text { Financiación prove- } \\
\text { niente de los accio- } \\
\text { nistas (propietarios) }\end{array}$} & Fuentes externas & $\begin{array}{l}\text { Acciones communes } \\
\text { Acciones privilegiadas }\end{array}$ \\
\cline { 2 - 3 } & Fuente Internas & $\begin{array}{l}\text { Utilidades retenidas (no distribuidas) } \\
\text { Decreto de dividendos en acciones }\end{array}$ \\
\hline \multirow{2}{*}{$\begin{array}{l}\text { Financiación prove- } \\
\text { niente de terceros }\end{array}$} & $\begin{array}{l}\text { Financiación de institucio- } \\
\text { nes financieras }\end{array}$ & $\begin{array}{l}\text { Préstamos a largo plazo } \\
\text { Préstamos hipotecarios } \\
\text { Arrendamiento financier }\end{array}$ \\
\cline { 2 - 3 } & $\begin{array}{l}\text { Emisión de títulos de deu- } \\
\text { da (obligaciones) }\end{array}$ & $\begin{array}{l}\text { Bonos } \\
\text { Bonos convertibles } \\
\text { Certificados de acciones (warrants) }\end{array}$ \\
\cline { 2 - 3 } & Otras fuentes & Arrendamiento operative \\
\hline
\end{tabular}

Fuente: Elaboración propia a partir de Vera (2009).

Para Gitman y McDaniel (2007), la deuda a largo plazo suele ser más costosa que la financiación a corto plazo debido a que existe una mayor incertidumbre respecto a la capacidad del prestatario para cumplir puntualmente con los pagos programados. Tal vez esa sea la causa de que (según Araujo, 2011), la mayoría de las pymes usan las utilidades retenidas como fuente de financiación, a través de lo cual, tienen la oportunidad de satisfacer sus necesidades de financiación con un método interno, el cual les permite una disminución de los costos que se incurren en comparación de solicitar financiación externo. 
Los principales tipos de deuda a largo plazo pueden ser con o sin garantía; entre los que tienen garantías están los préstamos con garantía prendaria o hipotecaria. También se tienen los bonos y el arrendamiento operativo y financiero. Van Horne y Wachowicz (2002) comentan que las obligaciones subordinadas y bonos de renta son las principales categorías de bonos sin garantía, mientras que los títulos hipotecarios son el tipo más común de instrumentos de deuda garantizada a largo plazo. Adicionalmente, se usan los arrendamientos de activos (contrato de uso del activo durante un tiempo determinado). Tanto el financiero (a largo plazo y no rescindible).

\section{d) Estrategias de financiación}

Apuntan Hax y Majluf (2004), que una estrategia es una respuesta a las oportunidades y amenazas externas, basada en las fortalezas y debilidades internas, para alcanzar ventajas competitivas. En este sentido, las estrategias financieras podrían definirse como aquellas decisiones desarrolladas para alcanzar ventajas competitivas, que involucran las finanzas de la empresa y responden a las oportunidades y amenazas del entorno. Persigue obtener recursos económicos en la cantidad, calidad y costo requeridos para que la organización pueda operar de manera sostenida, es decir, implica necesariamente, definir la estrategia de financiación. Debe definir si la financiación será a corto o a largo plazo, interno o externo, garantizado o no garantizado, si proviene de una institución pública o privada, luego dependiendo de esta decisión se diseña la estrategia financiera de la empresa.

Por lo tanto una estrategia de financiación debe analizar variables como: fuente (oferentes), plazo, instrumento, costo y garantías, las cuales estableciendo una analogía con los términos de la mercadotecnia constituiría la "Mezcla de Financiación" (Van Horne y Wachowicz, 2002).

En este sentido, Reyes y Díaz (2008) separan las estrategias en largo y corto plazo, dependiendo del efecto que se persiga con éstas. Para el largo plazo actúan sobre la inversión, la estructura financiera y la retención y/o reparto de utilidades. Mientras que para el corto plazo actúan sobre el capital de trabajo, la financiación corriente y la gestión del efectivo. Pero, en todo caso, siempre va a depender de la estrategia general del negocio y la selección de la mejor alternativa deberá seguir el criterio de maximizar el valor de la empresa. Continúan los autores explicando, que al actuar sobre la inversión puede orientarse al crecimiento (externo o interno) o a la desinversión y que deben utilizarse instrumentos de evaluación financiera como el valor actual neto (VAN), la tasa interna de rentabilidad (TIR), el índice de rentabilidad (IR) y el periodo de recuperación descontado (PRD, entre otros.

Cuando se actúa sobre la estructura financiera, el elemento de análisis será el mayor o menor riesgo financiero que estén dispuestos a aceptar. La deuda, por ejemplo, está exenta de impuestos y es relativamente económica, pero demasiadas deudas, incrementan el riesgo financiero y posibilidad de incumplimiento. Por otro lado, las estrategias basadas en la retención y/o reparto de utilidades, posibilitan el acceso a préstamos a largo plazo para financiar nuevas inversiones, entre otros aspectos. Esta estrategia se encuentra estrechamente vinculada con la de estructura financiera pues esta decisión tiene un impacto inmediato sobre la financiación permanente de la empresa, y provoca consecuentemente, variaciones en la estructura de las fuentes permanentes. 
Para el corto plazo, sostienen Reyes y Díaz (2008), la actuación sobre la administración del capital de trabajo, habitualmente obedece a la relación riesgo-rendimiento. En tal sentido, existen tres estrategias básicas: agresiva, conservadora e intermedia. La agresiva mantiene un capital de trabajo neto o fondo de maniobra relativamente pequeño debido a que los activos circulantes se financian exclusivamente con pasivos circulantes. La conservadora, significa que los activos circulantes se financian con pasivos circulantes y permanentes, manteniendo un alto capital de trabajo neto o fondo de maniobra. La estrategia intermedia contempla elementos de las dos anteriores, buscando un balance en la relación riesgo - rendimiento.

Sobre la financiación corriente, compuesto por fuentes espontáneas, bancarias y extra-bancarias, reporta un costo financiero que en dependencia de la fuente se presenta de forma explícita o no. De esta manera se puede apreciar que la definición de cómo deberá financiarse la empresa en el corto plazo responde a determinadas estrategias específicas, como es el caso del aprovechamiento del descuento por pronto pago, del ciclo de pagos que resulte adecuado a su vez a la estrategia de capital de trabajo o si estratégicamente conviene acudir al financiación externa. Al igual que el anterior, el criterio que priva en la selección es la mejor combinación riesgo - rendimiento.

Finalmente, sobre la gestión del efectivo, se hace énfasis en las políticas que deberán seguirse con los factores condicionantes de la liquidez de la empresa, a saber: los inventarios, los cobros y los pagos. En tal sentido, las acciones fundamentales con relación al efectivo son: 1 . Reducir el inventario tanto como sea posible, 2 . Acelerar los cobros tanto como sea posible 3. Retardar los pagos tanto como sea posible, sin afectar la reputación crediticia de la empresa, pero aprovechar cualquier descuento favorable por pronto pago. Gitman y McDaniel (2007) afirman deben medirse la efectividad de las acciones asociadas a la gestión del efectivo a través de: la razón rápida o prueba ácida, el ciclo de caja y/o la rotación de caja, el ciclo y/o la rotación de los cobros, el ciclo y/o la rotación de inventarios, así como el ciclo y/o la rotación de los pagos. Además, se recomienda la utilización del presupuesto de caja.

\section{Resultados}

En primer término se caracterizó el sector, para luego establecer las fuentes, estructura y estrategias de financiación del sector se suministros industriales de la ciudad de Maracaibo en Venezuela.

\section{a) Características de la pequeña y mediana empresa de suministros industriales del municipio Maracaibo}

La caracterización se hizo a través de siete indicadores que de alguna manera pueden dar una visión de estas organizaciones: número de trabajadores, ventas anuales, tiempo en el mercado, tipo de empresa, edad y nivel de estudio del gerente financiero, empleados temporales, mercados de compra y venta.

En cuanto al número de trabajadores, se encontró que el 75\% de las empresas tiene menos de 5 trabajadores y sólo el 25\% tiene entre 10 y 50 . Esto, de acuerdo con Katz (2009) implica que la mayoría son microempresas; sólo una cuarta parte puede calificarse de pequeñas empresas (Pérez, 2004). Sin embargo, a pesar de ser 
microempresas, sus ventas declaradas las sitúan en un rango de pequeñas empresas de acuerdo con los montos manejados por el DPDPMI ${ }^{3}$, lo cual da una idea de alta rentabilidad relativa.

Con respecto al tiempo de las empresas en el mercado, el 85\% tiene más de 10 años, lo cual implica que son empresas consolidadas, en edad madura, a decir de Pérez (2004). Siendo el 75\% empresas familiares (aquellas en las cuales una familia tiene más del 50\% de las acciones). A pesar de ser empresas maduras y familiares, los gerentes financieros son jóvenes o de mediana edad (95\% menores de 55 años, $65 \%$ menores de 40$)$ y $100 \%$ universitarios, incluso algunos con postgrado $(15 \%)$.

Esto es consistente con lo planteado por Pérez (2004) en cuanto a la juventud y preparación de los gerentes en las micro y pequeñas empresas. Por otra parte, se encontró un resultado que contradice el planteamiento de Pérez (2004), quien afirma que las pymes tienden a usar más empleados temporales para tener más flexibilidad ante lo incierto del mercado. Al analizar la proporción entre empleados fijos y temporales, se encuentra una relación 60 a 40, lo cual indica que más de la mitad del personal es fijo.

Otro aspecto que se consideró relevante, fue indagar sobre quiénes son sus proveedores y clientes. En este sentido, se encontró que sirven mayormente a los mercados local y estatal, y sólo una microempresa joven, se está enfocando en el mercado nacional. Lo contrario ocurre al analizar a sus proveedores, quienes en más del 85\% son internacionales y nacionales. Compran fuera, venden dentro.

Resumiendo los hallazgos (cuadro 3), puede decirse que el sector estudiado está conformado básicamente por microempresas sólidas, familiares, con trayectoria, dirigidas por personal profesional, funcionan con muy poco personal, sin embargo tienen ventas de empresas medianas, lo cual implica que son altamente rentables y bien administradas. No incursionan en mercados foráneos ya que tienen suficiente mercado local y regional, pero sus proveedores no son de la zona, lo cual implica que no promueven el desarrollo regional.

Cuadro 3. Características de las pymes de suministro industrial en Maracaibo

\begin{tabular}{|l|l|}
\hline \multicolumn{1}{|c|}{ Indicador } & \multicolumn{1}{c|}{ Característica resaltante } \\
\hline Número de trabajadores & $\begin{array}{l}\text { Microempresas (máximo cinco trabaja- } \\
\text { dores) }\end{array}$ \\
\hline Antigüedad & Entre 10 y 20 años \\
\hline Tipo de empresa según capital accionario & Familiares \\
\hline Edad y nivel de estudios del gerente financiero & Universitarios de menos de 40 años, \\
\hline Contratación de empleados temporales & $\begin{array}{l}\text { Mayor proporción de fijos (60 a } 40 \\
\text { aprox.) }\end{array}$ \\
\hline Ventas por mercado que sirve & Locales y regionales \\
\hline Compras por mercado proveedor & Nacionales e internacionales \\
\hline
\end{tabular}

Fuente: Elaboración propia a partir de la data recabada

Decreto $N^{\circ}$ 6.215, con Rango, Valor y Fuerza de Ley para la Promoción y Desarrollo de la Pequeña y Mediana Industria y Demás Unidades de Producción Social. 


\section{b) Fuentes de financiación utilizadas por el sector}

Se analiza en este apartado si las empresas estudiadas utilizan financiación a corto o a largo plazo, y específicamente, cuáles fuentes en cada uno. En cuanto a las fuentes de corto plazo espontáneas, se encontró que el $100 \%$ utiliza crédito de proveedores y pasivos acumulados y califican esta fuente como buena o muy buena en $95 \%$ de los casos. Van Horne y Wachowicz (2002), establece que ésta es una forma de deuda importante para el pequeño empresario puesto que al no tener respaldo, su utilización no amerita que las empresas ofrezcan garantías de activos y en la práctica es una financiación gratuita y rápida.

Con respecto al financiación bancario de corto plazo sin garantías, se encontró que en promedio, el 90\% utiliza esta forma de financiación. Pagarés, líneas de crédito y tarjetas de crédito empresariales (crédito revolvente) son utilizados masivamente por las organizaciones, sin preferencia por una en particular con una aceptación de muy bueno y bueno en un $95 \%$ de los casos.

Estos resultados, parecieran indicar que en el ámbito local, no se presentan los obstáculos planteados por el Banco Interamericano de Desarrollo (2002), cuando expresa que entre los obstáculos de las Pymes para acceder al crédito, desde el punto de vista de la demanda, se encuentran, el alto costo del crédito, la falta de confianza de los bancos respecto de los proyectos, el exceso de burocracia de los intermediarios financieros, y la petición de excesivas garantías. Pareciera que los planteamientos de Bloch y Granato (2007) según los cuales la oferta de crédito a Pymes se caracteriza por altas tasas de interés (el doble que para las grandes empresas), plazos cortos y exigencias elevadas de garantías, no es una realidad para estas empresas.

En relación con las fuentes extraordinarias de corto plazo (papeles comerciales, cartas de crédito y préstamos privados de accionistas: todas las empresas históricamente han utilizado cartas de crédito, aun cuando en la actualidad la situación país (niveles de insolvencia, control cambiario y ausencia de divisas) ha hecho que esta fuente esté congelada, mientras que los papeles comerciales y préstamos a accionistas se siguen utilizando. Sin embargo, las empresas reportan la satisfacción con esta fuente mayoritariamente como regular, incluso un $20 \%$ estima que los resultados son malos. Este resultado podría estar relacionado con lo señalado por Moori (2007) quien afirma que pese a que la carta de crédito goza de preferencia como fuente extraordinaria, algunas pymes no logran reunir todos los requisitos, por lo cual no siempre es considerado su resultado favorable.

Yendo a la financiación con garantías, se localiza el garantizado con inventarios y con cuentas por cobrar; en este caso se encontró, que las empresas no utilizan sus cuentas por cobrar y muy poco sus inventarios para obtener préstamos. Estos hallazgos contradicen los planteamientos de Algieri (2007), quien afirma que las cuentas por cobrar son garantías utilizadas por las Pymes a corto plazo, dentro de lapsos semestrales. De las cinco fuentes de financiación disponibles a corto plazo, las empresas estudiadas utilizan mayoritariamente las espontáneas y bancarias sin garantías.

En cuanto las fuentes de financiación de largo plazo, se tienen las proveniente de los accionistas (propietarios y nuevas emisiones de acciones) y provenientes de terceros (bancarias, obligaciones y arrendamiento). Se constató que las únicas fuentes de financiación a largo plazo que utilizan las empresas consultadas son las utilidades retenidas no distribuidas y en muy pocos casos, los dividendos. 
Mientras que ninguna empresa emite nuevas acciones para financiarse; ninguna usa financiación bancario a largo plazo, ni obligaciones (emisión de bonos) o arrendamiento. Lo anterior implica que los accionistas sacrifican sus utilidades en el corto plazo para el desarrollo de la empresa a mediano y largo, opción considerada con muy buenos resultados por quienes la utilizan. Estos hallazgos confirman el planteamiento de Araujo (2011) quien expresa que esta fuente interna tiene el doble propósito de satisfacer sus necesidades de financiación sin incurrir en los costos de solicitar financiación externo.

Resumiendo, se identificaron como fuentes de financiación básicas en estas empresas, el crédito comercial, los pasivos acumulados y la financiación bancaria a corto plazo. Mientras que a largo plazo utilizan las utilidades retenidas, no empleando la financiación a largo plazo proveniente de terceros, tal como se presenta en el cuadro 4.

Cuadro 4. Fuentes de financiación utilizadas

\begin{tabular}{|l|l|}
\hline \multicolumn{1}{|c|}{ Plazo } & \multicolumn{1}{c|}{ Fuentes utilizadas } \\
\hline \multirow{2}{*}{ Corto plazo } & Crédito comercial \\
& Pasivos acumulados \\
& Crédito bancario sin garantías \\
& Cartas de crédito \\
\hline Largo plazo & Utilidades retenidas \\
\hline
\end{tabular}

Fuente: elaboración propia a partir de la data recabada

\section{c) Estructura y estrategia de financiación del sector}

En atención a lo anterior, se pudo determinar la estructura de financiación de las pyme comercial de suministros industriales del Municipio Maracaibo, es decir, cuál es el uso de fuentes internas y externas de fondos. En este sentido, se indagó sobre el uso de la financiación externa de corto plazo, obteniéndose como respuesta en el $100 \%$ de los casos, que lo utilizan tanto para el activo circulante, como para el activo fijo. Lo anterior se constituye en una fuerte carga para estas organizaciones, al adquirir activos que reportarán beneficio en el mediano plazo y pagarlos con recursos de corto plazo. Se ratifica que no utilizan fuentes externas de financiación a largo plazo. De igual manera, los respondentes acotaron que utilizan su patrimonio (mayoritariamente utilidades retenidas y en menor medida, reservas) para financiar tanto activos circulantes (86\%) como activos fijos (14\%).

De los resultados anteriores, y las respuestas de los gerentes financieros, se concluye que la estructura de financiación de estas empresas es $20 \%$ externa y $80 \%$ interna. Los gerentes expresaron que si bien utilizan fuentes externas, sólo lo hacen a corto plazo y apenas sobrepasa el $20 \%$, mientras que el resto lo financian con fondos internos, bien sea a largo o a corto plazo, utilizando básicamente aumentos de capital, utilidades retenidas y en menor medida las reservas. Como se observa utilizan distintas opciones, en correspondencia con lo señalado por pero básicamente su financiación es interna.

Con respecto a las estrategias de financiación utilizadas por las pyme comercial de suministros industriales del Municipio Maracaibo, se indagó sobre las estrategias a largo plazo (sobre la inversión, la estructura financiera y las utilidades retenidas) 
y a corto plazo (sobre el capital de trabajo, la financiación corriente y la gestión de efectivo). Se encontró que el $90 \%$ de las empresas se perciben a si mismas en crecimiento y en ese sentido están ampliando el negocio. Sin embargo, expresan que no evalúan financieramente las inversiones a largo plazo. Lo anterior implica que no usan criterios técnicos de maximización del valor de la empresa en la toma de decisiones. Al no utilizar herramientas financieras para evaluar y en el entendido de que tienen que hacer alguna evaluación, se presume que es basada únicamente en la experiencia de los empresarios.

Por otra parte, al ser consultados sobre la posibilidad de tener financiación externa de largo plazo, el 100\% indicó una respuesta afirmativa. Las razones argumentadas se centraron en la imposibilidad de financiar el crecimiento de la empresa a través del aporte financiero de los socios o de la reinversión de utilidades, por cuanto la situación económica limita la disposición de recursos en forma oportuna. Vale decir en este punto que las empresas no tienen estrategias sobre la retención y/o reparto de utilidades. La razón de ello, la explicaron las unidades informantes indicando que la situación del país, limita las inversiones en el mismo, dadas las condiciones de incertidumbre, y no son utilizadas en este momento para la planificación a largo plazo.

En relación con las estrategias de corto plazo, específicamente el capital de trabajo, la mitad de las empresas financia los activos circulantes con pasivos circulantes, mientras que en la otra mitad la financiación es mixta (circulantes y permanentes, como capital social o reservas. De los resultados puede inferirse que sólo un $50 \%$ busca un cierto balance que luce razonable; aun cuando los actores no lo perciben como la búsqueda de un balance riesgo-rendimiento, de hecho implica la búsqueda del normal funcionamiento de la empresa con parámetros de liquidez aceptables, pero buscando a la vez que la participación de fuentes permanentes que propician lo anterior, no determine la presencia de costos excesivamente altos y con ello se pueda lograr un rendimiento total aceptable.

En cuanto a la financiación corriente, como se indicó anteriormente, se basa en cuentas y efectos por pagar, sueldos y salarios e impuestos y en menor medida (35\%) bancarios. Con respecto a la gestión de efectivo, el 100\% acelera cobros, retarda pago y aprovecha descuentos por pronto pago; aunque el $80 \%$ no utiliza razones financieras con las cuales monitorear la situación de la empresa. De hecho, la única razón utilizada por el $20 \%$ restante es la razón ácida, pero no manejan rotación de caja, cobros, inventarios o pagos y menos aun, un presupuesto de caja.

Resumiendo puede decirse que las las pymes estudiadas, se ubican en una etapa de crecimiento, utilizando como criterio ampliar el negocio. No evalúan financieramente las inversiones a largo plazo, consideran conveniente aumentar la financiación externo. En cuanto a las estrategias sobre el capital de trabajo, la mitad financia activos circulantes con pasivos circulantes, y la mitad utiliza también pasivos permanentes. En referencia a las estrategias de financiación corriente, incluyen las cuentas $\mathrm{y}$ efectos por pagar, salarios o sueldos, impuestos u otras retenciones, y fuentes bancarias; así como en la gestión de efectivo, aceleran los cobros, retardan los pagos y aprovechan los descuentos por pronto pago. 


\section{Conclusiones}

Las principales conclusiones de este trabajo, apuntan a que las empresas estudiadas (Pequeña y mediana empresa (pyme) comercial de suministros industriales del Municipio Maracaibo), son básicamente microempresas sólidas, en su mayoría de menos de cinco trabajadores, con empleados fijos, con capital accionario familiar, trayectoria entre 10 y 20 años, dirigidas por personal profesional de menos de 40 años, bien administradas y altamente rentables. No incursionan en mercados foráneos ya que tienen suficiente mercado local y regional, pero sus proveedores no son de la zona, lo cual implica que no promueven el desarrollo regional.

En cuanto a la financiación, estas empresas no se financian a largo plazo con terceros, sólo utilizan fuentes internas, hasta ahora, estos empresarios han preferido la financiación interna, básicamente aumento de patrimonio, utilidades retenidas y en menor medida reservas para todos sus activos fijos. No obstante, este criterio está cambiando ya que debido a la situación económica que limita la disposición de recursos, están considerando conveniente aumentar a la financiación externa a largo plazo (por necesidad, no por convicción). Como corolario del uso de las fuentes, la estructura de financiación es básicamente interna.

Adicionalmente, estas empresas se visualizan a sí mismas en etapa de crecimiento, con una clara orientación a ampliar el negocio. Sin embargo, la gran mayoría de estos comercios, no tiene estrategias financieras en el largo plazo, ni en cuanto a la inversión (no evalúa financieramente las inversiones a largo plazo, sólo utilizan el criterio de los directivos), ni en cuanto a la definición de la estructura o la utilización de las utilidades retenidas. Las condiciones de incertidumbre del país, mantienen a estas firmas con un horizonte de corto plazo. El largo plazo, el nivel estratégico, no está siendo considerado, aun cuando ellos están conscientes del efecto de esta actitud en sus finanzas.

Con respecto a las estrategias financieras a corto plazo, se nota una ausencia de técnicas de análisis para mejorar el uso del dinero; utilizan estrategias muy básicas, sólo la mitad combina la financiación de los activos circulantes con pasivos circulantes y pasivos permanentes. Solo un 35\% utiliza fuentes bancarias de financiación para el capital de trabajo y en su gestión del efectivo, si bien aprovechan los descuentos por pronto pago, no los ofrecen.

En términos generales, estas organizaciones son bastante cerradas a las influencias externas, hasta ahora han preferido mantenerse con un control financiero casi total, sin apalancamiento, evitando las deudas externas, lo cual a su vez limita su expansión. No innovan en cuanto a estrategias financieras y son más bien receptoras. A pesar de estar administradas por profesionales universitarios menores de 40 años, no utilizan técnicas de evaluación financiera o indicadores de gestión, ni razones financieras. Su alta rentabilidad en contraste con su pequeño tamaño, les ha reforzado las prácticas tradicionales y hasta ahora no han visto la necesidad de cambiarlas. Por otro lado, la alta incertidumbre legal, social y económica que vive el país los orienta hacia un horizonte de muy corto plazo, sin expectativas.

Podría concluirse que se comprueban los planteamientos de Bolaños (2003) en cuanto a que en la mayoría de las pymes familiares, las decisiones no se toman técnicamente, sino por criterio y carecen de formalidad en la planeación financiera. Sin embargo, no se pudo comprobar que el bajo uso del crédito bancario de las pymes estudiadas sea debido, como lo plantea el BID (2012), a problemas de oferta, mas 
bien, sugiere que estas organizaciones hasta ahora no han querido demandar recursos por no perder control de sus organizaciones o no querer dar información hacia afuera. Posiblemente, en el futuro esta situación cambie debido a la precaria situación del país que por ende, afecta también a estas empresas. Los resultados mencionados, parecieran desmitificar el lugar común de que las pymes tienen menos acceso al crédito que la gran empresa.

En atención a los resultados y conclusiones planteados, pareciera importante que las empresas del ramo, consideraran diversificar las fuentes de financiación, combinando un mejor uso de las fuentes. De esta manera modificarían la estructura actual de financiación combinado la financiación externa e interna para una optimización del gasto financiero. De igual manera, sería conveniente incorporar estrategias de inversión a largo plazo, así como el uso de instrumentos para la evaluación de las mismas, así el desarrollo rentable y armónico dependería menos de decisiones individuales y más de análisis técnico y del entorno.

\section{Bibliografía}

Algieri, M. (2007). Alternativas de Financiación para la Pequeña y Mediana Empresa (Pyme) de las Industrias Metálicas y Metalmecánicas. Especialización en Gerencia de Finanzas, Universidad Centroccidental "Lisandro Alvarado: Barquisimeto.

Álvarez, H. (2008). Proyecto: La estructura de financiación y el crecimiento de las pequeñas y medianas empresas. Facultad de Ciencias Económicas, Universidad Nacional de Córdoba. Argentina.

Araujo, P. (2011). Retención de Utilidades como Fuente de Financiación en las PYMES Industriales del Sector Carmen Sánchez de Jelambi del Municipio Valera Estado Trujillo. Departamento de Ciencias Económicas, Administrativas y Contables Universidad de Los Andes Núcleo Rafael Rangel: Trujillo

Aybar C., Casino, A. y López, J. (2001). Jerarquía de Preferencias y Estrategia Empresarial en la Determinación de la Estructura de Capital de la Pyme: Un Enfoque con Datos de Panel. Editor: Instituto Valenciano de Investigaciones Económicas, S.A. Primera Edición Marzo 2001: Valencia.

Banco Interamericano de Desarrollo, Bid (2012). Encuesta: 77\% de los bancos en América Latina y Caribe planean aumentar créditos a las pymes. Disponible en: http://www. iadb.org/es/noticias/comunicados-de-prensa/2012-11-19/encuesta-credito-para-las-pymes-2012-en-america-latina,10222.html . Accedido el 18/10/2016.

Bloch, R. y Granato, L. (2007). Las Pymes y el acceso al crédito [Página web en línea]. Revista Oidles Vol 1, $N^{\circ}$ 2. Disponible: www.eumed.net. Consultado el 06/09/2015.

Bolaños, R. (2003). La PYME, motor de desarrollo. Artículos Pyme. Disponible en: http:// pyme.com.mx/articulos_pyme/todoslosarticulos/la_pyme_motor_de_desarrollo.htm. Accedido el 06/09/2015

Brigham, E. y Houston, J. (2006). Fundamentos de Administración Financiera. Décima edición. Editorial Thomson Learning. México.

Briozzo, A. y Vigier, H. (2006). La Estructura de Financiación PYME. Papel de trabajo. Disponible en página web:http://mpra.ub.uni-muenchen.de/. Accedido el 06/09/2015

Chávez, N. (2006). Introducción a la investigación educativa. Editorial Graficas, S.A. Caracas.

Díaz, Y. y Piña E. (2013). Pymes en la actualidad: lo que debe conocer el gerente de inver- 
siones frente a la regulación jurídica del sistema financiero venezolano. CICAG, Vol. 10, No 1. Maracaibo.

Ferrero, C. (2011). Compilador. Eliminando barreras: El financiación a las pymes en América Latina. CEPAL, Naciones Unidad. Chile.

Guía de Asistencia Técnica (2007). Estructura Financiera del Capital de la Empresa. Financiación 2007, Mendoza Productiva, Gobierno de Mendoza, Argentina. Disponible en página web: www.portalpymes.mendoza.gov.ar. Accedido el 06/09/2015.

Gitman, L. y Mcdaniel, C. (2007). El futuro de los negocios. Quinta edición. Cengage Learning Editores, S.A. de C.V. México D.F.

Hax, A. y Majluf, N. (2004). Estrategias para el liderazgo competitivo. Primera edición. Ediciones Granica S.A. Argentina.

Hernández, R. Fernández, C., y Baptista, M. (2010) Metodología de la investigación. 5ta edición. Editorial Mc Graw- Hill. México

Hernández L, Romero Jenny, Portillo Rafael, Hernández Ridelis (2001). Las fuentes de financiación a corto plazo y su administración en la Pequeña y Mediana Industria (PYMI) en la región zuliana. (Sector confección, 1998-1999). Revista de Ciencias Sociales (RCS). Vol. Vii, No. 1, Enero-Abril 2001, Pp. 112-137. Faces - Luz Issn 1315-9518

Herrera, G. Limón, E. y Soto María (2005) Fuentes de financiación en época de crisis en $O b$ servatorio de la Economía Latinoamericana, Número 67, septiembre 2006, en http:// www.eumed.net/cursecon/ecolat/index.html. Accedido el 18/9/2015

Higuerey, A. (2004). Decisiones Financieras a Corto Plazo. Tema 7. Universidad de Los Andes. Núcleo Universitario "Rafael Rangel". Departamento de Ciencias Económicas y Administrativas, Trujillo.

Higuerey, A. (2006). Política de dividendos. Universidad de los Andes. Núcleo Rafael Rangel. Trujillo.

Katz, R. (2009). El papel de las TIC en el desarrollo. Propuesta de América Latina a los Retos Económicos Actuales. Colección Fundación Telefónica. Editorial Ariel, S.A. Barcelona. España

Moori, V. (2007). Características Del Financiación Bancario A Las Exportaciones De Pymes Industriales. Informe de la Comisión Económica para América Latina y el Caribe (1997-2007). Documento de Trabajo No. 132. Buenos Aires: Editado por la CEPAL.

Pérez, D. (2004). Análisis estratégico para el desarrollo de la pequeña y mediana empresa. Estado de Veracruz. Primera edición. Servicio de Publicaciones de la Universidad de Cantabria. México. Disponible en página web: www.books.google.co.ve . Accedido el $18 / 09 / 2015$.

Parra, J. (2006). Guía de Muestreo. Tercera edición. Facultad de Ciencias Económicas y Sociales de La Universidad del Zulia. Coordinación de Planificación. Colección XLVIII Aniversario FCES.

Pollack M. y García A. (2004). "Crecimiento, competitividad y equidad: rol del sector financiero. Serie financiación del desarrollo”. Cepal. United Nation Publications Business \& Economics. 82 pgs. Disponible en: https://books.google.co.ve/books?id=zauhbh0andoc\&printsec $=$ frontcover\&source $=$ gbs_ge_summary_r\&cad $=0 \# \mathrm{v}=$ onepage \&q\& $\mathrm{f}=$ false. Accedido el 19/10/2016

Ross, S.; Westerfield, R.; y Jaffe, J. (2005). Finanzas Corporativas. Séptima edición. McGraw-Hill Interamericana Editores, S.A. De C.V. México, D.F.

Rubio, F. (2006). Estructura de financiación: ¿cuánta deuda debería incorporar en mi empresa?. Documento del Munich Personal RePec Archive. Paper No. 578. Disponible en página web: http://mpra.ub.uni-muenchen.de/578. Accedido el 18/09/2015. 
Van Horne, J. y Wachowicz, J. (2002). Fundamentos de Administración Financiera. Undécima edición. Pearson Educación de México, S.A. de C.V. Naucalpan de Juárez, Edo. de México.

Vera, M. (2001). Gestion financiera de la pequeña y mediana industria de la ciudad de Maracaibo. En Revista de Ciencias Sociales. Vol. 7 No. 1. Abril 2001. Facultad de Ciencias Económicas y Sociales. Universidad del Zulia. Maracaibo. pp. 65-89

Vera, M. (2009). Estrategias Financieras Empresariales. Guía de estudio. Facultad de Ciencias Económicas y Sociales. División de estudios para graduados. Maestría en Gerencia de Empresas. Universidad de Zulia. Maracaibo, Venezuela. 